\title{
Risk prediction of cardiovascular surgery in Japanese patients
}

\author{
Takahiro Nishida, MD • Munetaka Masuda, MD
}

Received: 28 February 2011

(C) The Japanese Association for Thoracic Surgery 2011

In this issue of General Thoracic and Cardiovascular Surgery, Kurazumi et al. ${ }^{1}$ report that JapanSCORE, which has recently been established in Japan, might be superior to the logistic EuroSCORE for risk prediction of cardiac or thoracic aortic surgery in one Japanese university hospital.

Risk prediction of cardiovascular surgery is a topic of major interest, and risk stratification of surgical procedures using a superior algorithm would help improve the quality control of surgical care. Simple crude mortality figures are no longer sufficient to assess the quality control of treatment at institutions. Therefore, several algorithms have been developed to improve the accuracy of predicting the operative risk associated with cardiothoracic surgery.

Two major sophisticated databases have been developed for cardiovascular surgery: the STS National Database in the United States ${ }^{2}$ and the EuroSCORE system in Europe. ${ }^{3}$ Because of its simplicity in regard to evaluating the operative risk for individual patients, the EuroSCORE, especially the logistic EuroSCORE, ${ }^{4}$ has been widely used to predict mortality associated with cardiac surgery not only in European countries but also in Japan. ${ }^{5-8}$ However, the limitation of the STS National

This editorial refers to the article by Kurazumi et al. on pp. 599-604 of this issue of General Thoracic and Cardiovascular Surgery.

T. Nishida

Department of Cardiovascular Surgery, Kyushu University,

Fukuoka, Japan

M. Masuda $(\triangle)$

Department of Surgery, Yokohama City University, 3-9 Fukuura, Kanazawa-ku, Yokohama, Kanagawa 236-0004, Japan

Tel. +81-45-787-2644; Fax +81-45-786-0226

e-mail: mmasuda@yokohama-cu.ac.jp
Database and the EuroSCORE is that these algorithms have not been established for Japanese patients.

Thus, JapanSCORE was developed with the aim of establishing a Japanese original risk prediction model. It was accomplished by analyzing data from the Japan Adult Cardiovascular Surgery Database (JACVSD). The JACVSD was started in 2000 to estimate surgical outcomes after cardiovascular procedures in many centers throughout Japan. The database captured clinical information from approximately 180 hospitals $(32.7 \%$ of all Japanese units performing cardiac surgery) in 2005 and has increased the number to 302 hospitals in 2011 (http:// www.jacvsd.umin.jp/P5.html). The data collection form has a total of 255 variables (definitions are available online at www.jacvsd.umin.jp), which are almost identical to those in the STS National Database. Using these data from the JACVSD, three risk models-for isolated coronary artery bypass grafting (CABG), valve surgery, and thoracic aortic operations - have been established, as JapanSCORE. Superior risk stratification of isolated CABG, ${ }^{9,10}$ valve surgery, ${ }^{11}$ and thoracic aortic surgery ${ }^{12}$ has been achieved using this system.

It is well known that the operative mortality associated with cardiovascular surgery has improved because of technical modifications of the surgery. Thus, a risk prediction model should always be modified to improve the accuracy of its predictions. From this point of view, the additive EuroSCORE, originally established in 1999, was modified to become the logistic EuroSCORE in 2003 because the additive EuroSCORE tended to underestimate the operative risk for the high-risk group. To correct the underestimation in very high-risk patients and to perform full statistical comparisons with other systems with ease, comprehensive information on the logistic regression equation of the score was published. ${ }^{4}$ 
Hence, the logistic regression version of the score (the logistic EuroSCORE) could be calculated (http://www. euroscore.org/index.htm).

Although the STS Database and EuroSCORE are useful for evaluating operative risk, it might be not correct to apply these algorithms to Japanese patients. ${ }^{13}$ The difference in time for data collection might be a weak point of the EuroSCORE as compared with the JapanSCORE because the data for JapanSCORE were collected between 2000 and 2005, whereas the data for EuroSCORE were collected in 1995. It is thus obvious that the risk prediction model based on the Japanese database is a better model for evaluating the operative risk for Japanese patients. As Kurazumi et al. ${ }^{1}$ point out, JapanSCORE might be a better model than EuroSCORE because there are differences between the Japanese and people from Western countries in regard to genetics, the degree of mixing of racial backgrounds, education, workload, access to medical facilities, and social systems.

Finally, JapanSCORE, as the superior original risk prediction model, will produce many new findings on the topic of risk prediction and stratification and will help us improve the quality control of cardiovascular surgery in Japan. The Japanese Association of Thoracic Surgery publishes annual reports each year, but the reports are not based on risk-adjusted data. Risk-adjusted assessment of clinical results for each institution is essential for evaluating and comparing the quality of institutions precisely. This may improve the quality control of cardiovascular surgery in Japan. JapanSCORE is quite new and is still developing compared with the STS National Database and EuroSCORE, so the prediction of mortality and long-term results has not yet been established. Continuous system modifications based on continuing data collection from more institutions in Japan is mandatory to maintain the superior accuracy of JapanSCORE for risk prediction of cardiovascular surgery.

\section{References}

1. Kurazumi H, Mikamo A, Fukamitsu G, Kudou T, Sato M, Suzuki R, et al. Validation of the JapanSCORE versus the logistic EuroSCORE for predicting operative mortality of cardiovascular surgery in Yamaguchi University Hospital. Gen Thorac Cardiovasc Surg 2011;59:599-604.

2. Ferguson TB Jr, Dziuban SW Jr, Edwards FH, Eiken MC, Shroyer AL, Pairolero PC, et al. The STS National Database: current changes and challenges for the new millennium: Committee to Establish a National Database in Cardiothoracic Surgery, The Society of Thoracic Surgeons. Ann Thorac Surg 2000;69:680-91.

3. Nashef SA, Roques F, Michel P, Gauducheau E, Lemeshow $\mathrm{S}$, Salamon R. European system for cardiac operative risk evaluation (EuroSCORE). Eur J Cardiothorac Surg 1999;16: 9-13.

4. Roques F, Michel P, Goldstone AR, Nashef SA. The logistic EuroSCORE. Eur Heart J 2003;24:881-2.

5. Kawachi Y, Nakashima A, Toshima Y, Arinaga K, Kawano $\mathrm{H}$. Risk stratification analysis of operative mortality in heart and thoracic aorta surgery: comparison between Parsonnet and EuroSCORE additive model. Eur J Cardiothorac Surg 2001;20:961-6.

6. Kawachi Y, Nakashima A, Toshima Y, Arinaga K, Kawano $\mathrm{H}$. Evaluation of quality of cardiovascular surgery care using risk stratification analysis according to the EuroSCORE additive model. Circ J 2002;66:145-8.

7. Nishida T, Masuda M, Tomita Y, Tokunaga S, Tanoue Y, Shiose A, et al. The logistic EuroSCORE predicts the hospital mortality of the thoracic aortic surgery in consecutive 327 Japanese patients better than the additive EuroSCORE. Eur J Cardiothorac Surg 2006;30:578-82; discussion 582-3.

8. Hirose H, Noguchi $\mathrm{C}$, Inaba $\mathrm{H}$, Tambara $\mathrm{K}$, Yamamoto $\mathrm{T}$, Yamasaki M, et al. The role of EuroSCORE in patients undergoing off-pump coronary artery bypass. Interact Cardiovasc Thorac Surg 2010;10:771-6.

9. Motomura N, Miyata H, Tsukihara H, Okada M, Takamoto $\mathrm{S}$. First report on 30-day and operative mortality in risk model of isolated coronary artery bypass grafting in Japan. Ann Thorac Surg 2008;86:1866-72.

10. Saito A, Motomura N, Miyata H, Takamoto S, Kyo S, Ono M. Age-specific risk stratification in 13488 isolated coronary artery bypass grafting procedures. Interact Cardiovasc Thorac Surg 2011;12:575-80.

11. Motomura N, Miyata H, Tsukihara H, Takamoto S. Risk model of valve surgery in Japan using the Japan Adult Cardiovascular Surgery Database. J Heart Valve Dis 2010;19: 684-91.

12. Motomura N, Miyata H, Tsukihara H, Takamoto S. Risk model of thoracic aortic surgery in 4707 cases from a nationwide single-race population through a web-based data entry system: the first report of 30-day and 30-day operative outcome risk models for thoracic aortic surgery. Circulation 2008;118: S153-9.

13. Matsuura K, Ogino H, Matsuda H, Minatoya K, Sasaki H, Yagihara $\mathrm{T}$, et al. Limitations of EuroSCORE for measurement of risk-stratified mortality in aortic arch surgery using selective cerebral perfusion: is advanced age no longer a risk? Ann Thorac Surg 2006;81:2084-7. 